\title{
Enhancement of learning behaviour by a potent nitric oxide-guanylate cyclase activator YC-1
}

\author{
Wei-Lin Chien, ${ }^{1}$ Keng-Chen Liang, ${ }^{2}$ Che-Ming Teng, ${ }^{1}$ Sheng-Chu Kuo, ${ }^{3}$ Fang-Yu Lee ${ }^{3}$ and Wen-Mei Fu ${ }^{1}$ \\ ${ }^{1}$ Pharmacological Institute, College of Medicine, National Taiwan University, No. 1, Sec. 1, Jen-Ai Road, Taipei, Taiwan \\ ${ }^{2}$ Department of Psychology, National Taiwan University; Taipei, Taiwan \\ ${ }^{3}$ Graduate Institute of Pharmaceutical Chemistry, China Medical University, Taichung, Taiwan
}

Keywords: cGMP, learning, long-term potentiation, memory, NO, PKG, YC-1

\begin{abstract}
Memory is one of the most fundamental mental processes, and various approaches have been used to understand the mechanisms underlying this process. Nitric oxide (NO), cGMP and protein kinase G (PKG) are involved in the modulation of synaptic plasticity in various brain regions. YC-1, which is a benzylindazole derivative, greatly potentiated the response of soluble guanylate cyclase to NO (up to several hundreds fold). We have previously shown that YC-1 markedly enhances long-term potentiation in hippocampal and amygdala slices via NO-cGMP-PKG-dependent pathway. We here further investigated whether YC-1 promotes learning behaviour in Morris water maze and avoidance tests. It was found that YC-1 shortened the escape latency in the task of water maze, increased and decreased the retention scores in passive and active avoidance task, respectively. Administration of YC-1 30 min after footshock stimulation did not significantly affect retention scores in response to passive avoidance test. Administration of scopolamine, a muscarinic antagonist, markedly impaired the memory acquisition. Pretreatment of YC-1 inhibited the scopolamine-induced learning deficit. The enhancement of learning behaviour by YC-1 was antagonized by intracerebroventricular injection of NOS inhibitor L-NAME and PKG inhibitors of KT5823 and Rp-8-Br-PET-cGMPS, indicating that NO-cGMP-PKG pathway is also involved in the learning enhancement action of YC-1. YC-1 is thus a good drug candidate for the improvement of learning and memory.
\end{abstract}

\section{Introduction}

Learning and memory is one of the most intensively studied subjects in neuroscience. Various approaches have been used to understand the underlying cellular and molecular mechanisms (Abel \& Lattal, 2001; Schafe et al., 2001). Long-term potentiation (LTP) has been identified as a potential synaptic mechanism in several regions of the brain involved in learning and memory. Schaffer collateral inputs to pyramidal neurons in the hippocampus CA1 region exhibit a form of LTP that critically depends on $N$-methyl-D-aspartate (NMDA) receptor-mediated $\mathrm{Ca}^{2+}$ influx into the postsynaptic cell (Perkel et al., 1993; Tsien et al., 1996). Nitric oxide (NO) is widely used in neural circuits giving rise to learning and memory. $\mathrm{NO}$ is an unusual neurotransmitter in its modes of release and action. Nitric oxide participates in several types of synaptic plasticity, including long-term depression (LTD) in the cerebellum and striatum and LTP in the hippocampus and cerebral cortex (Daniel et al., 1998; Hawkins et al., 1998; Prast \& Philippu, 2001; Bon \& Garthwaite, 2003). It has been suggested that $\mathrm{NO}$, generated postsynaptically by $\mathrm{Ca}^{2+}$-calmodulindependent NO synthase (NOS), acts as a retrograde messenger (Son et al., 1996; Wilson et al., 1997). A major target of NO is soluble guanylate cyclase (sGC), which generates the intracellular second messenger cGMP. Consistent with a functional role of cGMP in the expression of LTP, sGC inhibitors suppress LTP (Zhuo et al., 1994; Boulton et al., 1995) and membrane-permeable dibutyryl-cGMP

Correspondence: Dr W.-M. Fu, as above.

E-mail: wenmei@ha.mc.ntu.edu.tw

Received 14 April 2004, revised 3 December 2004, accepted 23 December 2004 partially reverses the reduction of LTP by an NOS inhibitor (Haley et al., 1992). It is well known that cGMP regulates the activity of diverse proteins, including protein kinase $\mathrm{G}(\mathrm{PKG})$. The critical role of PKG was suggested by the finding that PKG inhibitors block LTP, and PKG activators facilitate LTP in response to rather weak tetanic stimuli (Zhuo et al., 1994).

A novel synthetic compound YC-1 [3-(5-hydroxymethyl-2-furyl)-1benzyl-indazole] has been shown to activate purified sGC and sensitizes the enzyme for NO in vitro, in human platelets, and in smooth muscle cells (Ko et al., 1994; Mulsch et al., 1997; Friebe et al., 1998). YC-1 produced an enormous stimulation of the sensitivity of the purified enzyme to NO up to several hundredfold (Friebe et al., 1996). We previously found that YC-1 enhanced LTP in hippocampal Schaffer collateral-CA1 synapse via a NO-cGMP-PKGdependent pathway (Chien et al., 2003). In addition, YC-1 also potentiated LTP induction in the amygdala (Chien et al., 2003). Here we found that YC-1 greatly improved rodent learning behaviour when examined with the Morris water maze and avoidance tests.

It is known that the central cholinergic systems play an important role in learning and memory. The cholinergic deficit is a prominent hallmark of Alzheimer's disease (Palmer, 1996). It was found that the decrease of acetylcholine release may be responsible for memory disturbances (Aprikyan \& Gekchyan, 1988). Here we further investigated the effects of $\mathrm{YC}-1$ on memory disturbances induced by the antagonist of cholinergic receptors, scopolamine. It was demonstrated that $\mathrm{YC}-1$ is also able to inhibit the scopolamineinduced memory deficits. These in vivo findings suggest a therapeutic potential of $\mathrm{YC}-1$ as a drug candidate for improving learning and memory. 


\section{Materials and methods}

\section{Electrophysiology}

Transverse slices of hippocampus $(400-\mu \mathrm{m}$ thick) were prepared from adult Wistar rats $(150-250 \mathrm{~g})$. The rats were killed by cervical dislocation and decapitated. Slices were immediately placed in icecold cutting buffer containing (in $\mathrm{mM}$ ): Slices were immediately placed in ice-cold cutting buffer containing (in $\mathrm{mM}$ ): $\mathrm{NaCl}, 124$; $\mathrm{KCl}, 3 ; \mathrm{Na}_{2} \mathrm{HPO}_{4}, 1 ; \mathrm{NaHCO}_{3}, 25 ; \mathrm{CaCl}_{2}, 0.5 ; \mathrm{MgSO}_{4}, 5$; glucose, 10 ; saturated with $95 \% \mathrm{O}_{2}$ and $5 \% \mathrm{CO}_{2}$. Slices were then maintained in an interface chamber at room temperature, where they were subfused with artificial cerebrospinal fluid (ACSF) containing (in $\mathrm{mM}$ ): $\mathrm{NaCl}, 124 ; \mathrm{KCl}, 4.4 ; \mathrm{Na}_{2} \mathrm{HPO}_{4}, 1 ; \mathrm{NaHCO}_{3}, 25 ; \mathrm{CaCl}_{2}, 2$; $\mathrm{MgSO}_{4}, 1$; glucose, 10 ; bubbled with $95 \% \mathrm{O}_{2}$ and $5 \% \mathrm{CO}_{2} . \mathrm{A}$ bipolar tungsten-stimulating electrode was placed in the middle of the stratum radiatum in the CA1 region, and extracellular field potentials were recorded using a glass microelectrode (5-10 M , filled with $2 \mathrm{M} \mathrm{NaCl}$ solution). The pulse duration was $100 \mu \mathrm{s}$, and test responses were elicited at $0.05 \mathrm{~Hz}$. The perfusion rate of ACSF was $1-2 \mathrm{~mL} / \mathrm{min}$.

\section{Behavioural tasks}

Morris water maze

Wistar rats (200-300 g) were used. Animals were housed individually and maintained on a $12 \mathrm{~h} / 12$ light/darkness cycle. All rats were allowed free access to food and water. The Morris water maze was performed in a circular pool (224 cm in diameter, $46 \mathrm{~cm}$ in height) located in a room with distinctive visual cues. Water was filled to a depth of $36 \mathrm{~cm}$ and a transparent plastic platform $(25 \times 25 \mathrm{~cm}, 32 \mathrm{~cm}$ in height) was located at the centre of a fixed quadrant. Training started by acclimating the rat to the task environment with 2 days of free-swimming in the pool with no platform. Each session lasted for $2 \mathrm{~min}$ and the rat was picked up from the pool by the experimenter. Rats received four consecutive daily training trials for the following 4 days. In each trial, the rat was placed into the water randomly from one of the quadrants. The rat had to swim until it climbed onto the platform submerged underneath the water. The time duration from entering the water to climbing onto the platform was recorded and defined as escape latency. If the rat failed to find the platform by $120 \mathrm{~s}$, it placed onto the platform by the experimenter. The rat stayed on the platform for $60 \mathrm{~s}$, which also served as the interval of trial. At the termination of each day's training, the rat was dried with towels and an electric heater before being placed into its home cage. On the seventh day, the probe test was performed. The platform was not placed in the tank and the rats were allowed to swim freely for $120 \mathrm{~s}$. The total time that subjects spent in each quadrant of the tank was recorded.

YC-1 $(1 \mathrm{mg} / \mathrm{kg})$ or vehicle was injected (i.p.) $10 \mathrm{~min}$ before the first trial. L-NAME $(1 \mu \mathrm{mol}$ in $10 \mu \mathrm{L} / 100 \mathrm{mM})$ or PKG inhibitors of KT5823 $(0.2 \mathrm{nmol}$ in $10 \mu \mathrm{L} / 20 \mu \mathrm{M})$ and Rp-8-Br-PET-cGMPS ( $1 \mathrm{nmol}$ in $10 \mu \mathrm{L} / 0.1 \mathrm{~mm}$ ) were administered by intracerebroventricular (i.c.v.) injections $20 \mathrm{~min}$ before the first trial. The cannula was positioned under anaesthesia $(200 \mathrm{mg} / \mathrm{kg}$ trichloroacetaldehyde) $1.0 \mathrm{~mm}$ lateral to the midline, $1.5 \mathrm{~mm}$ caudal to bregma, and $3.5 \mathrm{~mm}$ below the surface of the skull, according to the atlas of Paxinos \& Watson (1997). Cannulae were made of 23-guage stainless steel tubing, with a $0.33-\mathrm{mm}$ inner diameter and a $0.63-\mathrm{mm}$ outer diameter, to a length of $10 \mathrm{~mm}$. Dental cement was used to secure the cannula to two screws inserted in the skull. Rats were kept warm until recovery from anaesthesia. They recuperated for at least 1 week before being subjected to behavioural testing.
Passive avoidance and active avoidance

Rats were trained and tested on a one-trial step-through passive inhibitory avoidance task with a procedure modified from that described by Liang et al. (1998). Briefly, the apparatus was a trough-shape alley divided by a sliding door into a well-lit safe compartment and a dark shock compartment. On day 0 designated as context acclimation, the rat was placed into the lit side facing away from the door. As the rat turned around, the door was opened. It was allowed to enter the dark compartment for three times without receiving any foot-shock. The rat was then retrieved and returned to its home cage. On day 1 designated as foot-shock training, the rat was placed into the lid side and allowed to enter the dark site as in the previous day. After the rat stepped into the dark site, the door was closed and the rat received an inescapable foot-shock via a constant current shocker controlled by a timer (Lafayette Instruments, Model 80240 and Model 58010, IN, USA). After administration of the footshock $(0.6 \mathrm{~mA}, 0.6 \mathrm{~s})$, the rat was removed from the alley and returned to its home cage. In a retention test given on days 2, 3 or 11 , the rat was reintroduced into the alley and the latency to enter the shock compartment was taken as the retention score. No foot-shock was administered on the retention test, and testing was terminated at $600 \mathrm{~s}$.

For the active avoidance task, the rat was placed facing the door in the dark compartment of the alley described above. The door was lowered halfway to form a hurdle such that the rat could leap over the door into the lit compartment. Any rat that spontaneously crossed into the lit side within $10 \mathrm{~s}$ was excluded from this experiment. The footshock was turned on $10 \mathrm{~s}$ after the door was lowered; the rat had to escape into the lit compartment and the door was closed. The retention test was administered $24 \mathrm{~h}$ later. The latency to leave the dark compartment was measured as the retention score.

\section{Startle test}

Startle response to stimulus was determined using a standard startle chamber (San Diego Instruments, San Diego, CA, USA). The animal was hold in a Plexiglas cylinder tube (length $20 \mathrm{~cm}$, diameter $10 \mathrm{~cm}$ ) with a vibration sensor attached to the base. Each tube was encoded in a ventilated sound-attenuating cabinet (length $38 \mathrm{~cm}$, width $38 \mathrm{~cm}$, and height $55 \mathrm{~cm}$ ) that was dark, ventilated and sound attenuated. The acoustic startle stimuli were high-intensity white noise bursts delivered by a speaker $30 \mathrm{~cm}$ above the animal. The shock stimuli were squarewave direct currents generated from a programmable shocker (TI 30; Coulburn Instruments, San Diego, CA, USA). Scrambled shocks were delivered to a grid floor consisting eight metal rods inserted inside the cylinder. Motion of the animal within the cylinder was detected via vibration sensor for a period of $200 \mathrm{~ms}$ after initiation of the stimulus. The vibration was transduced into voltage, then the output of the transducer was led to a computer for sampling. The startle amplitude of each trial is defined as the maximal vibration within the $200 \mathrm{~ms}$.

After receiving injections of $0.1 \%$ dimethylsulphoxide (DMSO) in saline or YC-1, rats were held in their cage for $15 \mathrm{~min}$ and then placed into the startle apparatus with a continuous $55 \mathrm{~dB}$ background noise. After a 5-min acclimation period, 45 trials were presented with a $30-\mathrm{s}$ intertrial interval (ITI). Two types of stimuli were dispensed to elicit startle: One type of stimuli contained nine different intensities of electric shocks that were ranging from 0 to $1.6 \mathrm{~mA}$ with increments of $0.2 \mathrm{~mA}(0.1 \mathrm{~s})$. The other type of stimuli was white noise bursts that were composed by 10 noise bursts ( $40 \mathrm{~ms}$ in duration) with 30 -s ITIs of each of three intensities $(95,105$ and $115 \mathrm{~dB})$.

Each session contained 3 blocks of trials, and each block consisted of two different series. Each series was composed of six acoustic trials 
(two trials at each level.) followed by nine shock trials (one trial at each shock intensity). Different intensities for each stimuli modality were presented in a semi-random order within the separated phases of series. The total time elapsed for a test session was 28 min including the acclimation periods. At the end of the startle session, the rats were returned to their home cages. Average transducer output just prior to noise burst was saved as a baseline (VStart). In addition, the computer found the maximum startle amplitude within each of the samples (VPeak) and this value was also saved. Peak startle amplitude was expressed as VPeak - VStart for analysis.

The water maze and startle test were analysed using two-way analysis of variance (ANOVA) followed by the post hoc Scheffe test. The avoidance responses were analysed by the nonparametric Kruskal-Wallis test followed by the Mann-Whitney $U$-test. All protocols complied with institutional guidelines and were approved by Animal Care Committees of Medical College, National Taiwan University. This study adhered to guidelines for care and use of Experimental Animals of the National Science Council, R.O.C.

\section{Drugs used}

L-NAME, scopolamine, KT5823 and Rp-8-Br-PET-cGMPS were obtained from Sigma-Aldrich (St. Louis MO, USA); YC-1 was provided by Yung-Shin Pharmaceutical Industry Co. Ltd. (Taichung, Taiwan).

\section{Results}

\section{Concentration-dependent enhancement of LTP by YC-1}

We first examined the effect of YC-1 on the induction of LTP in Schaffer collateral-CA1 pathway of rat hippocampal slices. As shown in Fig. 1A, two trains of extracellular stimulation of Schaffer collaterals at $100 \mathrm{~Hz}$ for $1 \mu \mathrm{s}$ induced LTP in the synaptic inputs to CA1 pyramidal cells. Perfusion of YC-1 $(5.3 \mu \mathrm{M})$ for $6 \mathrm{~min}(3 \mathrm{~min}$ before and 3 min after weak tetanus) markedly increased the extent of LTP. YC-1 potentiated LTP in a concentration-dependent manner (Fig. 1B).

\section{Effects of YC-1 on learning behaviour}

Morris water maze

Substantial evidence implicates the hippocampus in acquisition and retention of spatial memory (Barnes, 1988). We then examined the effect of YC-1 on the acquisition of spatial memory in rats, using the Morris water maze. The rats received four training trials each day. YC-1 was injected (i.p., $1 \mathrm{mg} / \mathrm{kg}$ ) $10 \mathrm{~min}$ before the first trial of each daily training session. As shown in Fig. 2A, YC-1 shortened the escape latency of trials $2-4$ on the first training day, suggesting that $\mathrm{YC}-1$ promotes spatial memory. The escape latencies of trials $2-4$ were $93.8 \pm 8.7 \mathrm{~s}, 79.3 \pm 12.3 \mathrm{~s}$ and $57.6 \pm 12.5 \mathrm{~s}$ for control rats and $50.4 \pm 10.2 \mathrm{~s}, 47.3 \pm 6.8 \mathrm{~s}$ and $30.3 \pm 10.3 \mathrm{~s}$ for the YC-1-treated group, respectively $(n=10)$. The escape latencies of the first trial on the following three training days were also markedly shortened by YC-1. The escape latencies were $91.1 \pm 9.8 \mathrm{~s}, 47.1 \pm 3.9 \mathrm{~s}$ and $35.3 \pm 6.1 \mathrm{~s}$ for control rats and $55.4 \pm 12.8 \mathrm{~s}, 15.3 \pm 2.1 \mathrm{~s}$, and $13.4 \pm 1.7 \mathrm{~s}$ for the YC-1-treated group, respectively $(n=10)$. These results demonstrate that long-term memory is markedly improved by YC-1 administration. The summarized results are shown in Fig. 2B. The escape latency of the first trial is shown in this figure. The escape latency is persistently shorter in $\mathrm{YC}$-1-treated group throughout the

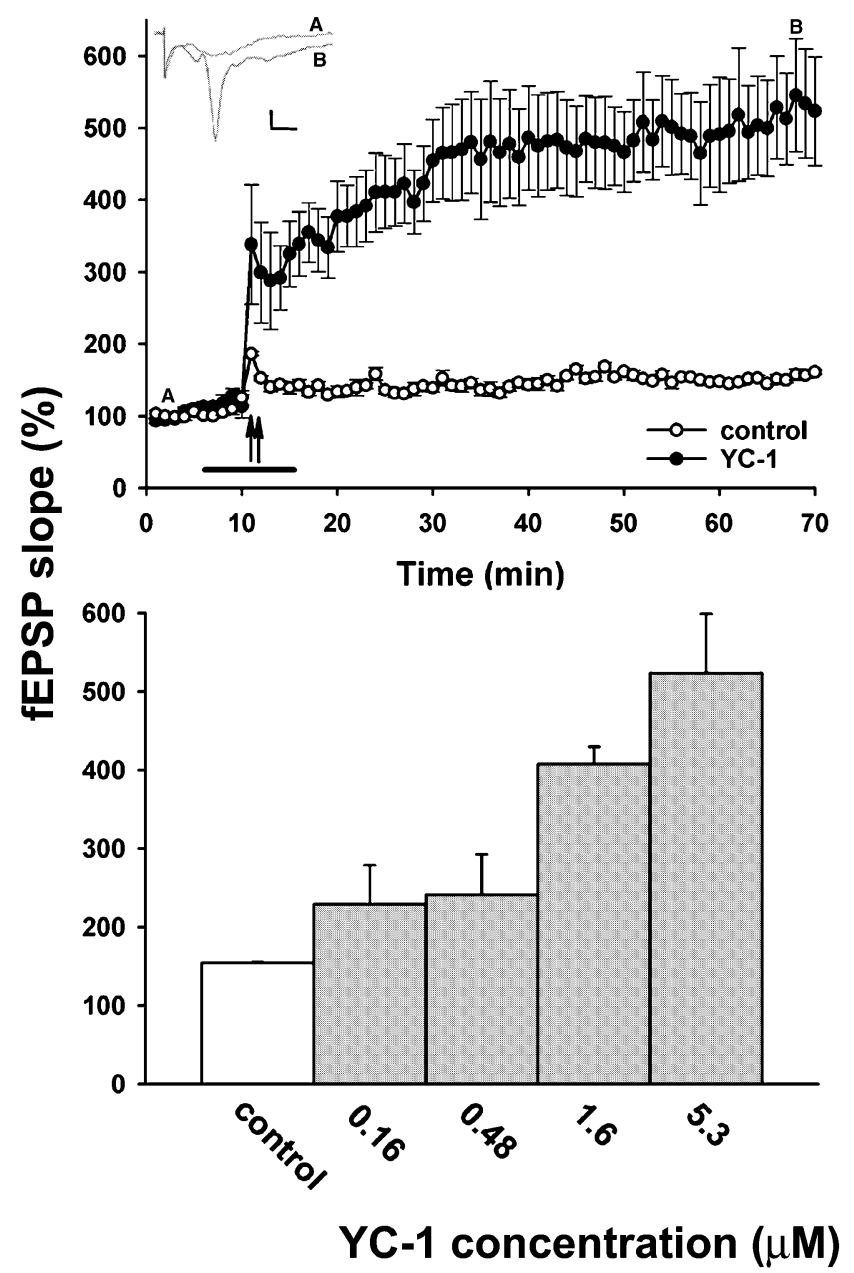

FIG. 1. Concentration-dependent enhancement of LTP by YC-1 in hippocampus. (A) Hippocampal slices were stimulated in the Schaffer collateral-CA1 pathway at a basal stimulating rate at $0.05 \mathrm{~Hz}$. LTP was induced by strong tetanus $(100 \mathrm{~Hz}$ for $1 \mathrm{~s}$, each separated by periods of $20 \mathrm{~s})$. YC-1 $(5.3 \mu \mathrm{M})$ given $3 \mathrm{~min}$ before and $3 \mathrm{~min}$ after strong tetanus potentiated the induction of LTP. The concentration-dependent effect of YC-1 is shown in $\mathrm{B}(n=4-$ 8 , mean \pm SEM).

whole experimental period up to 21 days, although YC-1 was only administered for initial 4 consecutive days. The mean escape latencies on day 21 were $13.5 \pm 1.8 \mathrm{~s}$ and $6.0 \pm 0.5 \mathrm{~s}(n=10$ each $)$ for the control and YC-1-treated rats, respectively. We further examined the effect of oral intake of YC-1 on the performance of the rats in the water maze task. The rats were given YC-1 mixed with $1.25 \%$ carboxymethylcellulose $(10 \mathrm{mg} / \mathrm{kg})$ orally $30 \mathrm{~min}$ before the first trial for six consecutive days. Similar to intraperitoneal administration of YC-1, oral intake of YC-1 also enhanced the spatial memory, as shown by the escape latency of the first trial in each daily training session (Fig. 2C).

As the NO-cGMP-PKG pathway is involved in the LTP-potentiating action of YC-1 (Chien et al., 2003), we then investigated the in vivo effect of L-NAME, KT5823 and Rp-8-Br-PET-cGMPS on the improvement of learning and memory by $\mathrm{YC}-1$. In these experiments, L-NAME $(1 \mu \mathrm{mol})$, KT5823 $(0.2 \mathrm{nmol})$ or Rp-8-Br-PET-cGMPS $(1 \mathrm{nmol})$ was administered i.c.v. $10 \mathrm{~min}$ before $\mathrm{YC}-1$ administration (i.p., $1 \mathrm{mg} / \mathrm{kg}$ ). As shown in Fig. 3, the NOS inhibitor L-NAME significantly blocked the enhancing effect of YC-1 on learning and memory. Intracerebroventricular administration of the PKG inhibitors 


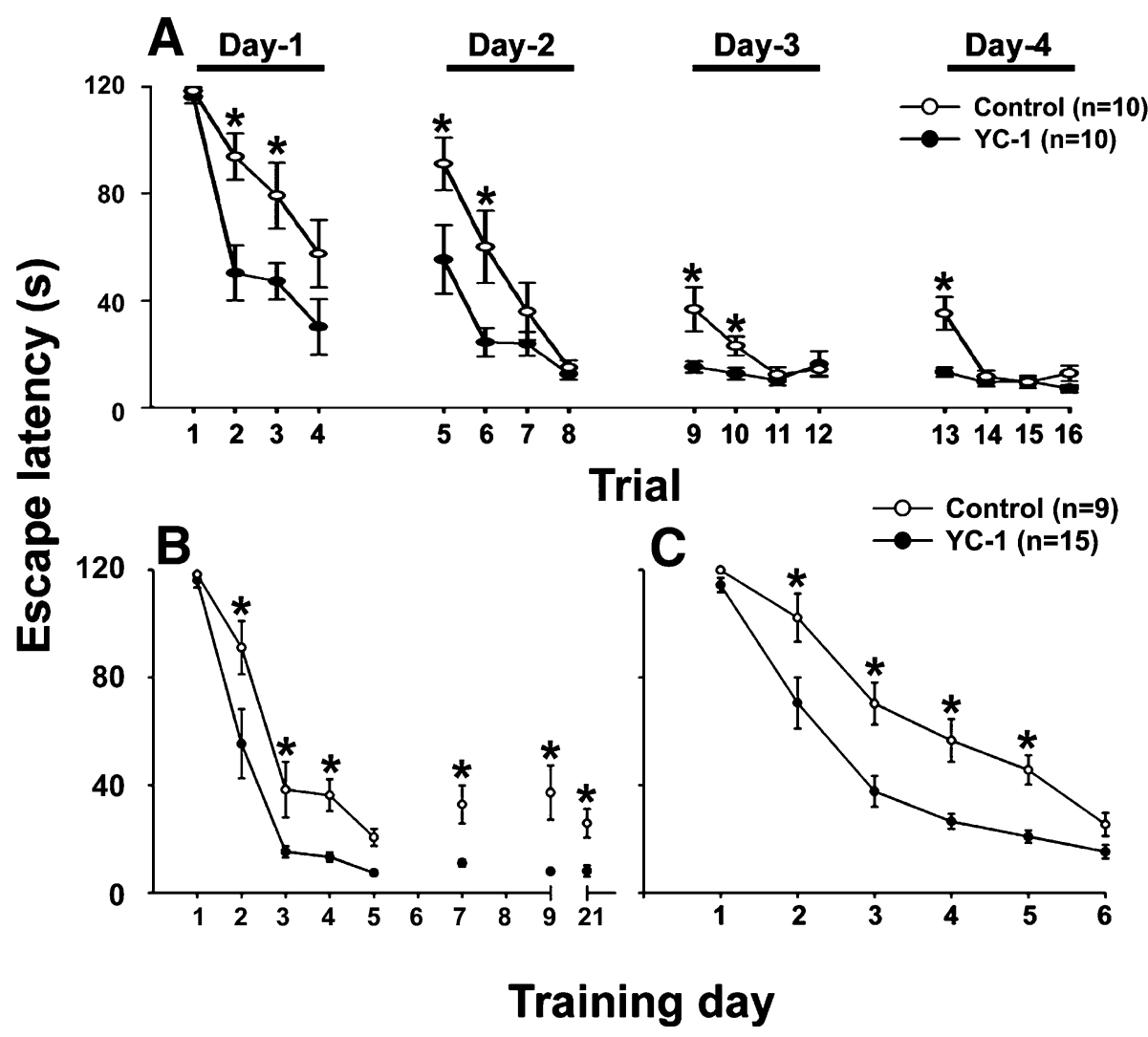

FIG. 2. Enhancement of water maze task by YC-1. YC-1 was injected (i.p., $1 \mathrm{mg} / \mathrm{kg}$ ) $10 \mathrm{~min}$ before the first trial of each daily training session. Note that YC-1 shortened the escape latencies of trials $2-4$ on the first day and of the first trial on the following 3 training days (A). (B) Each point represents the escape latency of the first trial of each daily training session. Note that YC-1 shortened the escape latency throughout the whole experimental period until day 21 although YC-1 was only given on the initial four consecutive days. ( $n=9$ and 10 for control and YC-1, respectively). (C) YC-1 was mixed with CMC (1.25\%) and administered orally $(10 \mathrm{mg} / \mathrm{kg}) 30 \mathrm{~min}$ before the first trial of daily training. Note that oral intake of YC-1 still enhanced water maze learning. Each point represents the escape latency of the first trial of each daily training session ( $n=9$ and 15 for control and $\mathrm{YC}-1$, respectively). ${ }^{*} P<0.05$ compared with control using two-way ANOVA followed by the post hoc Scheffe test. Open circles show control; closed circles show YC-1.

KT5823 and Rp-8-Br-PET-cGMPS also attenuated the memory enhancement caused by YC-1 (Fig. 3, ANOVA test among three groups: $F_{3,41}=49.2, P<0.001$ for Fig. $3 \mathrm{~A}$ and $F_{3,41}=29.2$, $P<0.001$ for Fig. 3B). It seems that L-NAME and KT5823 exert greater inhibition approximately throughout training on days 2-5. These results suggest that the potentiation of spatial memory by YC-1 is sensitive to inhibition by NOS and PKG inhibitors. Intracerebroventricular administration of L-NAME, KT5823 or Rp-8-Br-PETcGMPS alone also slightly exerted inhibition of learning behaviours as shown in the escape latency of first trial (Fig. 4A) or the mean escape latency of four trials (Fig. 4B) in the daily training sessions.

\section{Passive and active avoidance tasks}

Fear memory requires the participation of neurons in the amygdala (Cahill \& McGaugh, 1990; Gilbert et al., 1991). There is also evidence that $\mathrm{NO}$ is involved in activity-induced synaptic plasticity in some amygdaloid nuclei (Bernabeu et al., 1995; Watanabe et al., 1995; Telegdy \& Kokavszky, 1997). Because we found that YC-1 also enhanced LTP induction in the amygdala (Chien et al., 2003), we therefore investigated the effect of $\mathrm{YC}-1$ on the fear memory using passive avoidance test. As shown in Fig. 5A, rats received YC-1 (i.p., $1 \mathrm{mg} / \mathrm{kg}) 10 \mathrm{~min}$ before foot-shock training exhibited prolonged retention latencies in the safe compartment. The retention scores remained at a high level even if the rats were tested on day 11. More rats had retention latencies $>10 \mathrm{~min}$ in the $\mathrm{YC}$-1-treated group (5 out of 27 in the control group and 20 out of 24 in the YC-1-treated group, respectively) tested on both day 2 and day 11 . When YC-1 was mixed with $1.25 \% \mathrm{CMC}$ and delivered orally, the drug also greatly improved retention in the task. The mean retention scores on the day 2 test were $157.1 \pm 64.3 \mathrm{~s}$ and $369.2 \pm 70.2 \mathrm{~s}(n=10$ for each), respectively, for the control ( 2 out of 10 were longer than $5 \mathrm{~min}$ ) and YC-1-treated group ( 8 out of 10 were longer than $5 \mathrm{~min}$ ). A similar treatment with YC-1 was also effective in improving learning and memory in aged rats (1 year old) although the effect was much less pronounced than that in younger rats (data not shown).

The results shown above indicate that $\mathrm{YC}-1$ can prolong the retention of a passive avoidance response in rats. In this task, $\mathrm{YC}-1$ preferentially affects the formation of new memory, a process conceived to be related to the induction phase of LTP. The fear memory of passive avoidance relies on a brief experience of footshock. Consistent with the idea that NO, if generated by this training experience, is quickly metabolized, we found that YC-1 did not exert any effect on rat avoidance behaviour when it was injected $30 \mathrm{~min}$ after foot-shock (Fig. 5B). In addition, the enhancement effect of YC-1 could be antagonized significantly by L-NAME and KT5823 (i.c.v., $1 \mu \mathrm{mol}$ and $0.2 \mathrm{nmol}$, respectively; Fig. 5C). These results suggest that YC-1 can improve learning behaviours only when it was administered shortly before or during the learning experience. More interestingly, $\mathrm{YC}-1$ given $10 \mathrm{~min}$ before the context acclimation phase in which no foot-shock was administered also prolonged the retention 

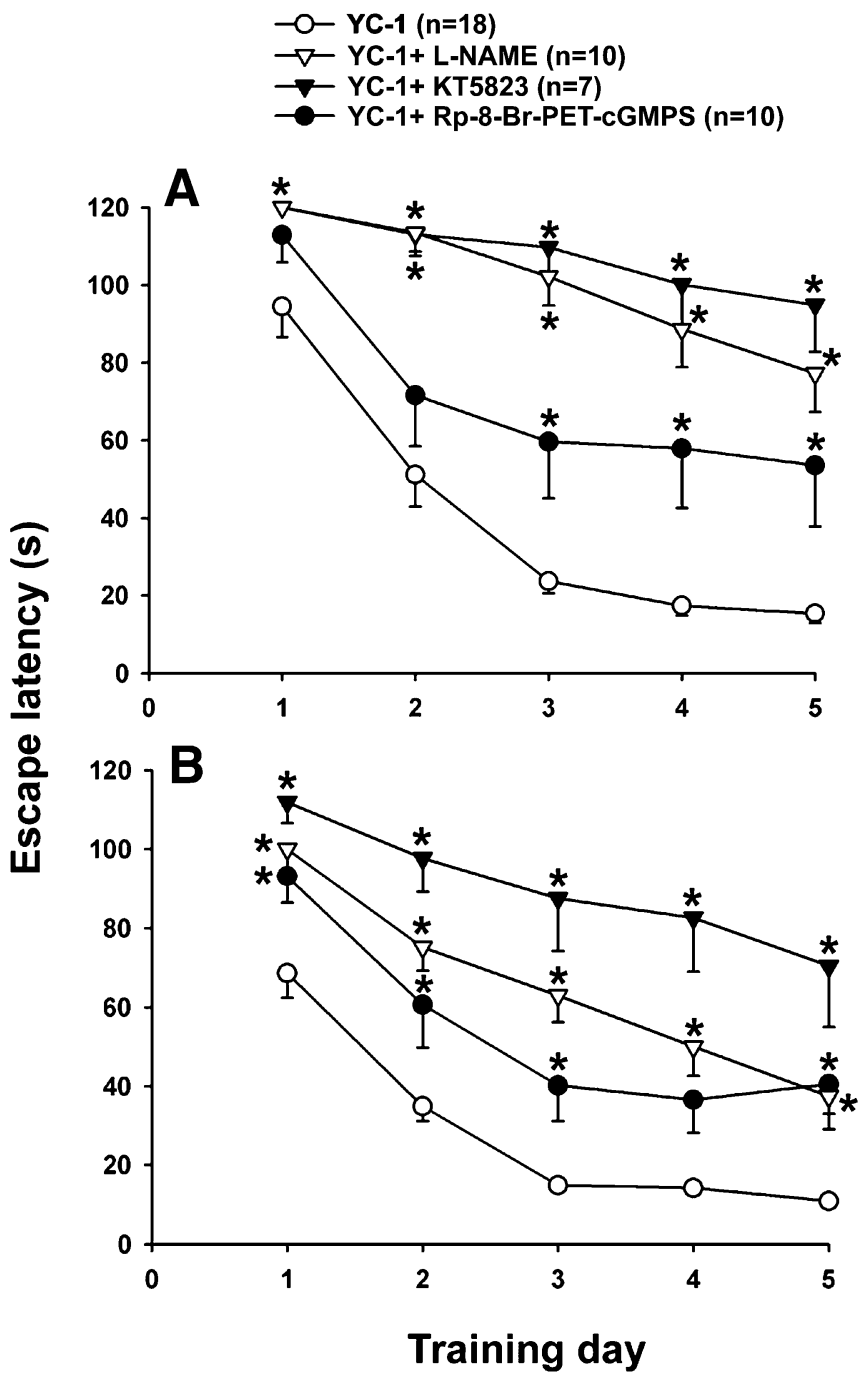

FIG. 3. Antagonism of L-NAME, KT5823 and Rp-8-Br-PET-cGMPS on the enhancement of learning by YC-1.YC-1 (i.p., $1 \mathrm{mg} / \mathrm{kg}$ ) was simultaneously injected with L-NAME (i.c.v., $1 \mu \mathrm{mol}$ ), KT5823 (i.c.v., $0.2 \mathrm{nmol}$ ), Rp-8-BrPET-cGMPS (i.c.v., $1 \mathrm{nmol}$ ) or saline $10 \mathrm{~min}$ before the first trial of daily training. Note that L-NAME, KT5823 and Rp-8-Br-PET-cGMPS impaired the enhancing effects of $\mathrm{YC}-1$ on the first trial (A) and the mean escape latency of four trials (B) of each daily training session. The number of animals tested per group was indicated in parenthesis. ${ }^{*} P<0.05$ compared with control using two-way ANOVA followed by the post hoc Scheffe test.

latencies in the test if foot-shock was administered on the subsequent day (Fig. 5D), suggesting that $\mathrm{YC}-1$ is capable of enhancing an otherwise latent memory for environmental cues.

For the active avoidance task, rats were put into the dark compartment. Foot-shock was administered $10 \mathrm{~s}$ later in motivating the rat to shuttle into the lit side. Retention was tested 2 days later. Injection of YC-1 10 min before foot-shock caused a marked improvement in retention performance by decreasing the escape latency from the shock-compartment to the safe compartment (Fig. 6). Furthermore, the memory enhancing effect of $\mathrm{YC}-1$ can also be effectively inhibited by L-NAME and KT5823 (i.c.v., $1 \mu \mathrm{mol}$ and $0.2 \mathrm{nmol}$, respectively). Results above show that YC-1 significantly improves learning and memory of both passive and active avoidance responses through a NO-cGMP-PKG signalling pathway in rats. We observed no change in startle response after injection of $\mathrm{YC}-1$ as assessed by foot-shock as well as acoustic stimulations (Fig. 7). Oral

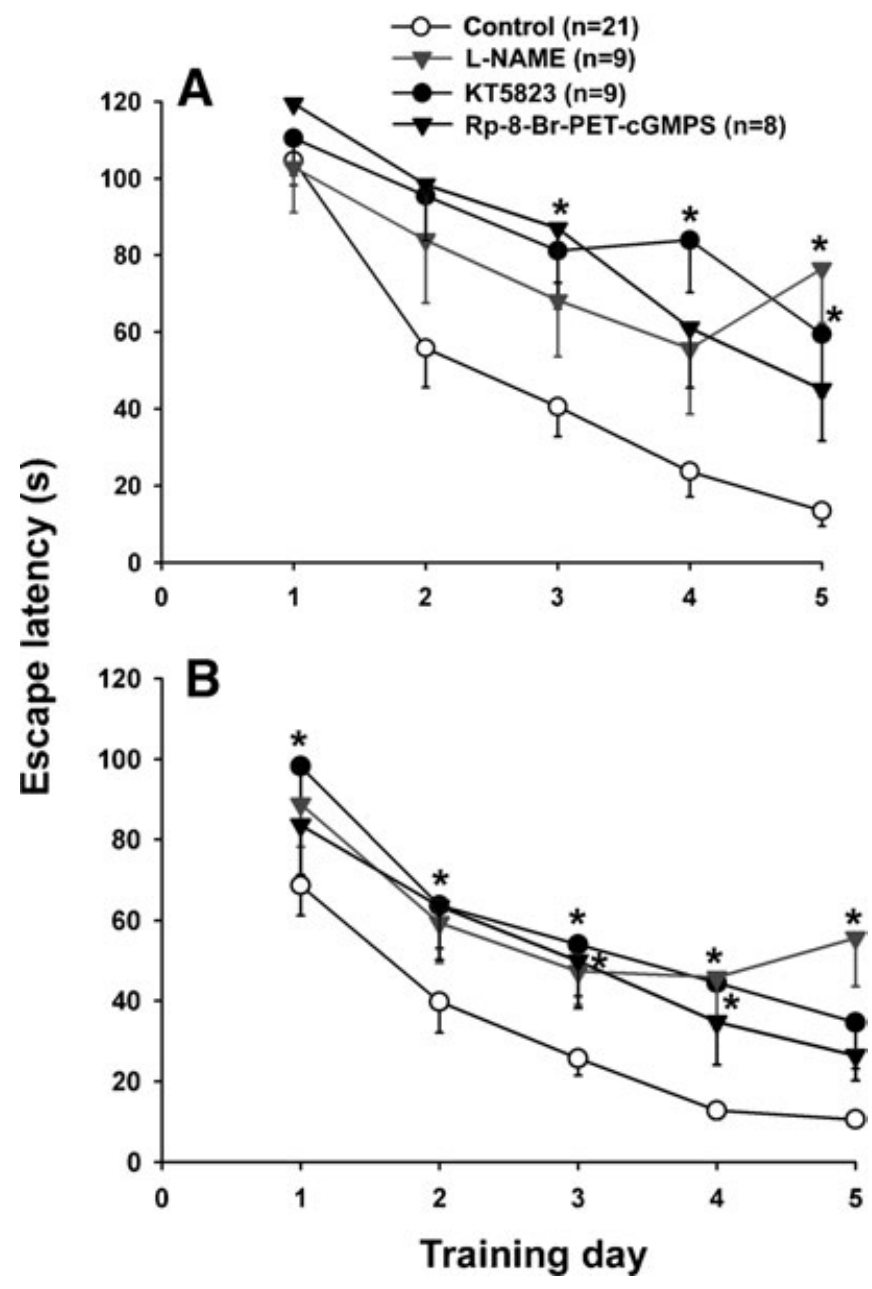

FIG. 4. Inhibition of water maze task by intracerebroventricular administration of L-NAME, KT5823 and Rp-8-Br-PET-cGMPS. L-NAME (1 $\mu \mathrm{mol})$, KT5823 (0.2 nmol), Rp-8-Br-PET-cGMPS (1 nmol) or saline was i.c.v. applied $10 \mathrm{~min}$ before the first trial of daily training. Each point represents the escape latency of the first trial (A) or the mean escape latency of 4 trials (B) of each daily training session. Note that L-NAME, KT5823 or Rp-8-Br-PET-cGMPS administration slightly impaired the learning behaviour. The number of animals tested per group was indicated in parenthesis. $* P<0.05$ compared with control using two-way ANOVA followed by the post hoc Scheffe test.

administration of YC-1 (10 mg/kg per day) for 3 weeks also did not affect the withdrawal latency on the hot-plate thermal $\left(55^{\circ} \mathrm{C}\right)$ test (control, $14.3 \pm 2.3 \mathrm{~s}, n=9$; YC-1, $12.9 \pm 3.8 \mathrm{~s}, n=10$ ). These results suggest that the enhancement of fear memory by $\mathrm{YC}-1$ does not result from the increase of shock sensitivity.

\section{Effect of YC-1 on scopolamine-induced impairment in learning behaviour}

In the Morris water maze test, administration of scopolamine $(0.2 \mathrm{mg} / \mathrm{kg}$, i.p. $20 \mathrm{~min}$ before the first trial of each daily training session) impaired learning abilities significantly. YC-1 (1 mg/kg, i.p) given concomitantly, antagonized the scopolamine-induced impairment of learning abilities on the performance in the Morris water maze. The escape latencies of the first trial (Fig. 8A) and the mean value of the four trials (Fig. 8B) during the six training days were markedly prolonged by the scopolamine treatment. YC-1 treatment significantly improved the learning. Figure $8 \mathrm{C}$ shows the probe test on day 7. The swimming paths were recorded by a camera and the data 


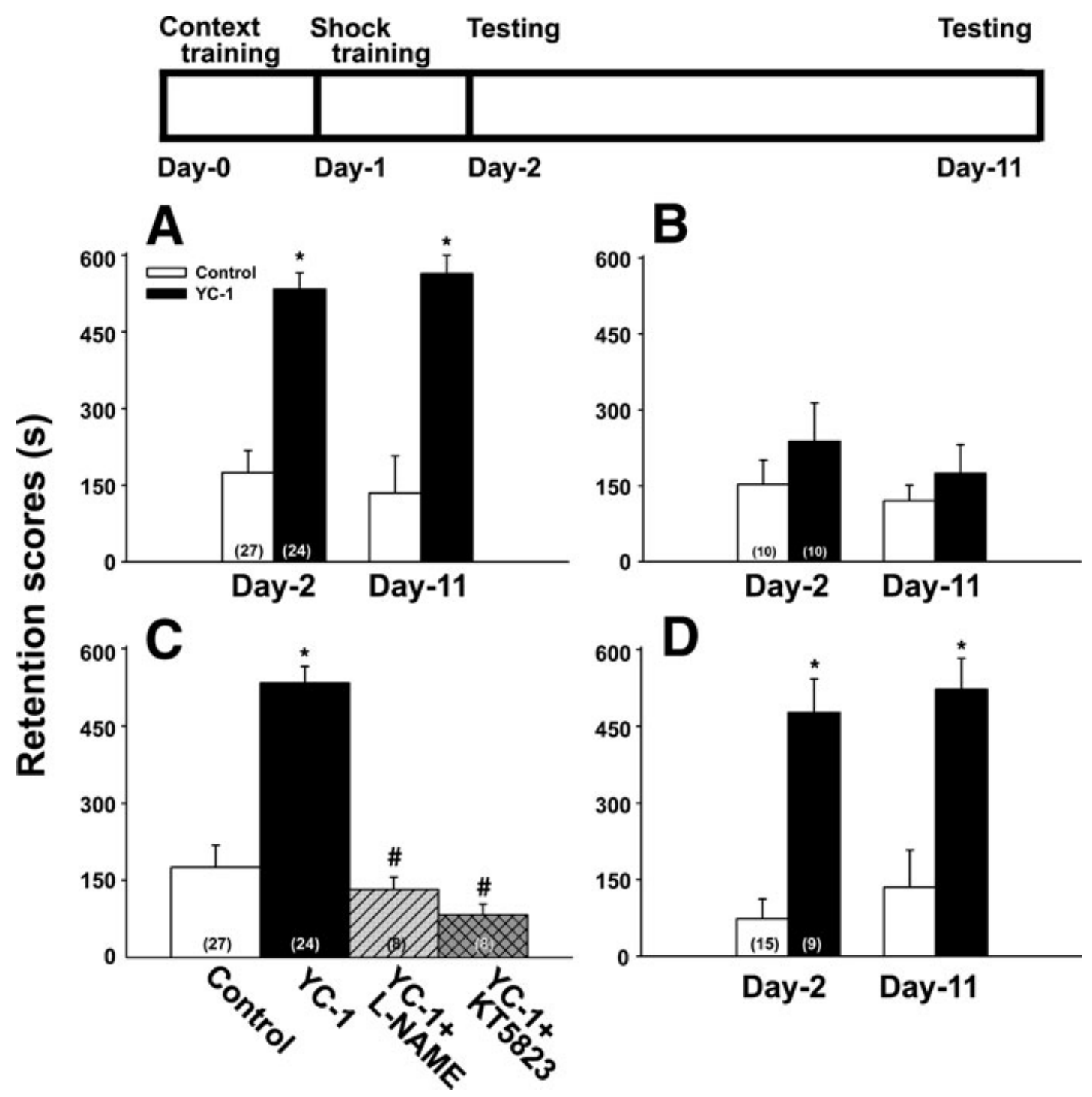

FIG. 5. Effects of YC-1 on passive avoidance tasks. Passive avoidance was assessed on day 2 and day 11 after training by measuring the latency to re-enter a chamber previously paired with foot-shock $(0.6 \mu \mathrm{A}, 0.6 \mathrm{~s})$. Administration of $\mathrm{YC}-1(1 \mathrm{mg} / \mathrm{kg}$, i.p.) $10 \mathrm{~min}$ before (A) but not $30 \mathrm{~min}$ after (B) footshock markedly prolonged the retention latency tested on both day 2 and day 11. (C) L-NAME (i.c.v., 1 umol) and KT5823 (0.2 nmol) antagonized the memory enhancing effect of YC-1 (i.p.) in the passive avoidance task when the rats were tested $48 \mathrm{~h}$ after the shock. (D) Rats received YC-1 (1 mg/kg, i.p.) $10 \mathrm{~min}$ before the context acclimation phase also prolonged the retention latencies in the passive avoidance task. Controls, open bars (A, B, D); filled bars, YC-1 treatment. Data are presented as mean \pm SEM $(n) .{ }^{*} P<0.05$ compared with control using nonparametric Kruskal-Wallis followed by Mann-Whitney $U$-test. $\# P<0.05$ compared with YC-1 + saline group.

were analysed on computer. All three groups showed bias towards the plate probe, and scopolamine significantly reduced the time on the plate probe. Concomitant administration of $\mathrm{YC}-1$ reversed the scopolamine-induced impairment on the probe test. The upper panels show the representative swimming path of the three groups.

We also examined the effect of YC-1 on scopolamine-induced impairment in passive avoidance test. As shown in Fig. 9, scopolamine given $20 \mathrm{~min}$ before the foot-shock training markedly decreased the retention scores of passive avoidance test. Concomitant administration of YC-1 significantly antagonized the scopolamine-induced impairment.

\section{Discussion}

Nitric oxide synthase is widespread in the nervous system and NO is thought to play important roles in activity-dependent synaptic plasticity and is involved in several types of learning and memory (Hawkins, 1996; Prast \& Philippu, 2001). In the present study, we have demonstrated that YC-1 improves learning and memory of rodents in a variety of behavioural tests through a NO-dependent pathway. We previously found that weak tetanic stimulation at $50 \mathrm{~Hz}$ for $0.5 \mathrm{~s}$ did not induce LTP unless YC-1 is present (Chien et al., 2003). In neurons, NO synthesis is stimulated by $\mathrm{Ca}^{2+}$ influx, which is induced by activation of glutamate receptors, from both NMDA and metabotropic receptors (Chien et al., 2003). Nitric oxide operates primarily as an intercellular messenger. It diffuses rapidly and influences NO-responsive target cells probably within spatial limits of approximately $0.3-0.4 \mathrm{~mm}$ (Lancaster, 1997). This property of NO allows YC-1 to enhance LTP in an input-specific manner and its influence on behaviour is therefore experience-dependent. It seems that many effects of NO are mediated by cGMP. Activation of the sGC, increase of cGMP formation and activation of PKG has been 


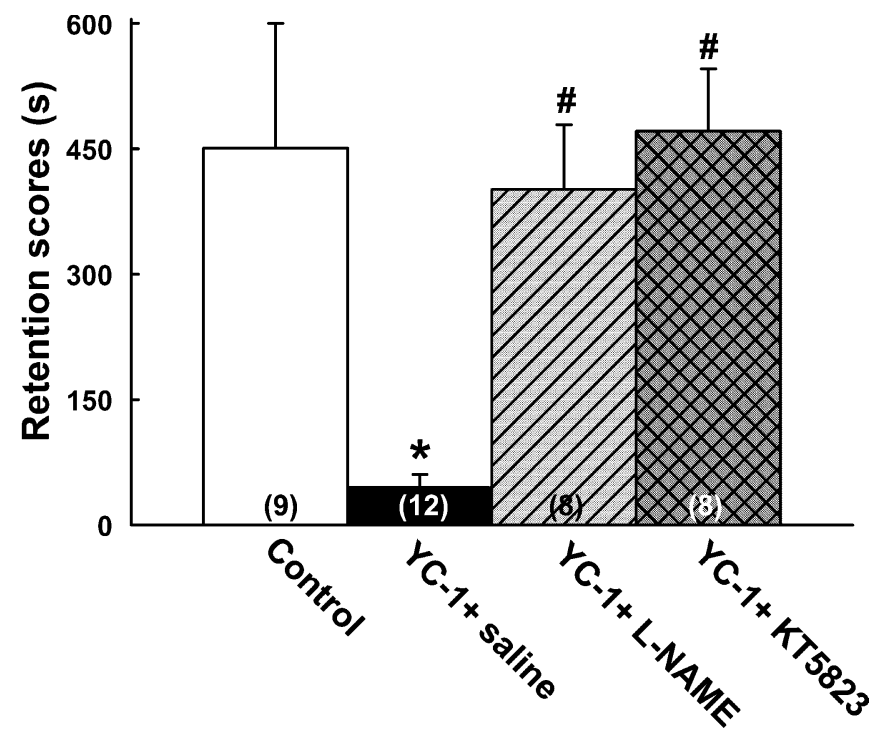

FIG. 6. Effects of YC-1 on active avoidance tasks. Active avoidance was assessed $24 \mathrm{~h}$ after training by measuring the latency of escaping from a compartment previously paired with foot-shock $(0.6 \mathrm{~mA}, 0.6 \mathrm{~s})$. Note that YC-1 shortened the retention latency in the active avoidance task. L-NAME (i.c.v., $1 \mu \mathrm{mol})$ and KT5823 $(0.2 \mathrm{nmol})$ also antagonized the memory enhancing effect of YC-1 (i.p.) in the active avoidance task. Data are presented as mean $\pm \operatorname{SEM}(n) .{ }^{*} P<0.05$ compared with control using nonparametric Kruskal-Wallis followed by the Mann-Whitney $U$-test. $\# P<0.05$ compared with $\mathrm{YC}-1+$ saline group.

suggested as the main signal transduction pathway of NO. Stimulation of neuronal cGMP synthesis might modulate synaptic functions and lead to the early phase of LTP (Hawkins et al., 1993, 1998). The NOcGMP-PKG pathway might also contribute to the late phase of LTP by causing induction of immediate early genes through phosphorylation of CREB (Gudi et al., 1996). CREB phosphorylation and gene induction are thought to contribute to the late, protein synthesisdependent phase of hippocampal LTP (Bourtchouladze et al., 1994; Carew \& Sutton, 2001), which may involve presynaptic as well as postsynaptic changes (Bernabeu et al., 1995; Bolshakov et al., 1997).

The relation between LTP and memory is a focus of intensive investigation (Shors \& Matzel, 1997; Malenka \& Nicoll, 1999). In the present study, administration of YC-1 greatly improved learning and memory in several tasks involving different brain structures including hippocampus, cerebral cortex and amygdala. We found that YC-1 did not affect memory retention when administered $30 \mathrm{~min}$ after footshock training, suggesting the action of YC-1 occurs during or immediately following the training. The present study also showed that YC-1 consistently improves memory in tasks with opposite motor demands (passive and active avoidance) and in tasks relying on different sorts of sensory and motivational stimuli. The startle scores in response to foot-shock or acoustic stimuli are not affected by $\mathrm{YC}-1$ treatment and the ability of YC-1 to enhance learning in the passive avoidance task when given in the context acclimation phase, suggesting that the memory improvement by YC-1 might not result from altering shock sensitivity.

NOS inhibitors reduce the capability of treated animals to acquire or retain information in several learning tasks (Prast \& Philippu, 2001). Here we reported that intracerebroventricular injection of L-NAME, KT5823 and Rp-8-Br-PET-cGMPS results in amnesia on later tests and severely impaired the enhancing effects of $\mathrm{YC}-1$ on long-term memory formation. NO is known to be required for consolidation of new memory into long-term storage in different behavioural tasks ( $\mathrm{Li}$ et al., 1995). It is also required for LTD induction in cerebellar slices because NOS inhibitors block LTD (Ito, 2000). Furthermore, cGMP and PKG have also been shown to produce activity-dependent longlasting effects in cerebellum and sensory-motor cortex (Ito \& Karachot, 1990; Shibuki \& Okada, 1991). Therefore, our results provide further evidence that NO-cGMP-PKG is an important signalling pathway for synaptic plasticity and the consolidation of new memory. YC-1 potentiated the response of $\mathrm{sGC}$ to $\mathrm{NO}$ and enhanced LTP induction and memory acquisition in various kinds of learning tasks. It has been reported that $\mathrm{YC}-1$ at higher concentrations might inhibit phosphodiesterase (Friebe et al., 1998; Galle et al., 1999). Therefore, the slight inhibition of phosphodiesterase by lower concentrations of YC-1 used in the present study can not be excluded and may partially contribute to the increase of cGMP. The downstream effectors or transcription factors regulated by PKG are likely to be involved in memory consolidation, and their identities remain to be elucidated. We previously found that incubation of un-stimulated hippocampal slices with YC-1 $(1.6 \mu \mathrm{M})$ plus the NO donor nitroprusside increased the immunofluorescence and protein expression of pERK and pCREB in pyramidal neurons, which were inhibited by the PKG inhibitor KT5823 (Chien et al., 2003). It appears that improvement of memory by $\mathrm{YC}-1$ could result from the direct effect of YC-1 on synaptic plasticity but not through cerebral vasodilation. In addition, it needs higher concentrations of YC-1 to cause vasodilation (Galle et al., 1999).
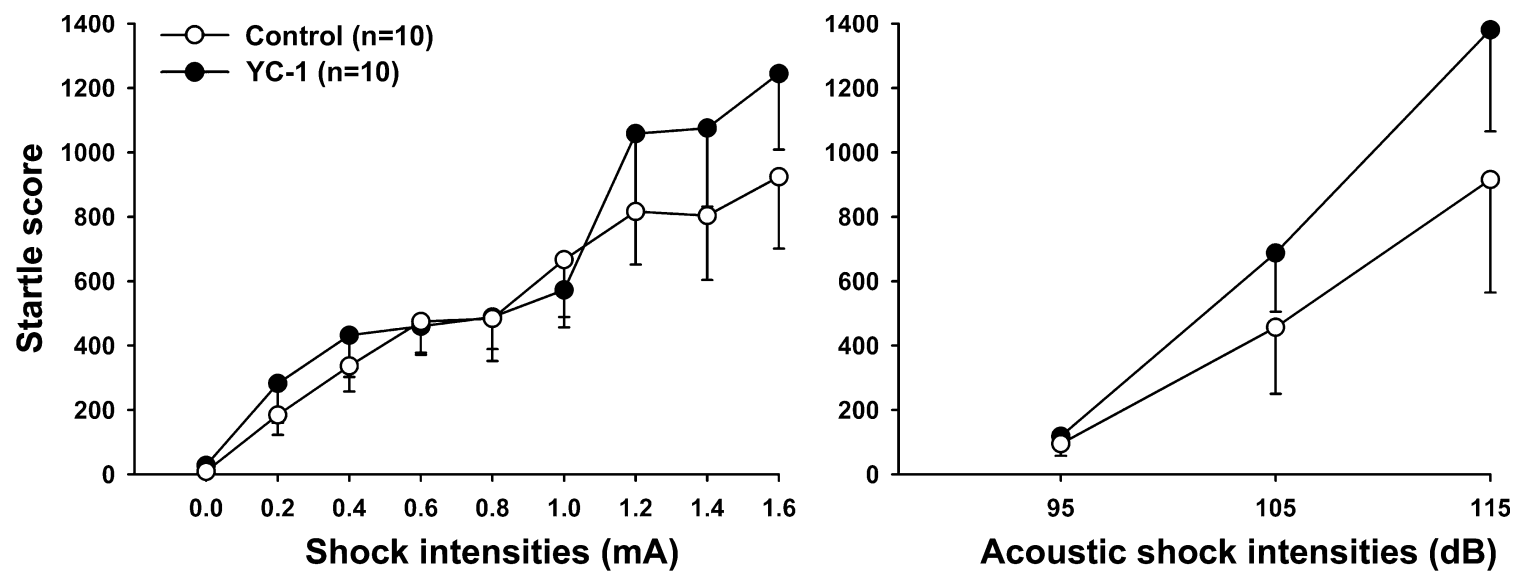

FIG. 7. The lack of effect of YC-1 on shock startle (left panel) and acoustic startle (right panel). 

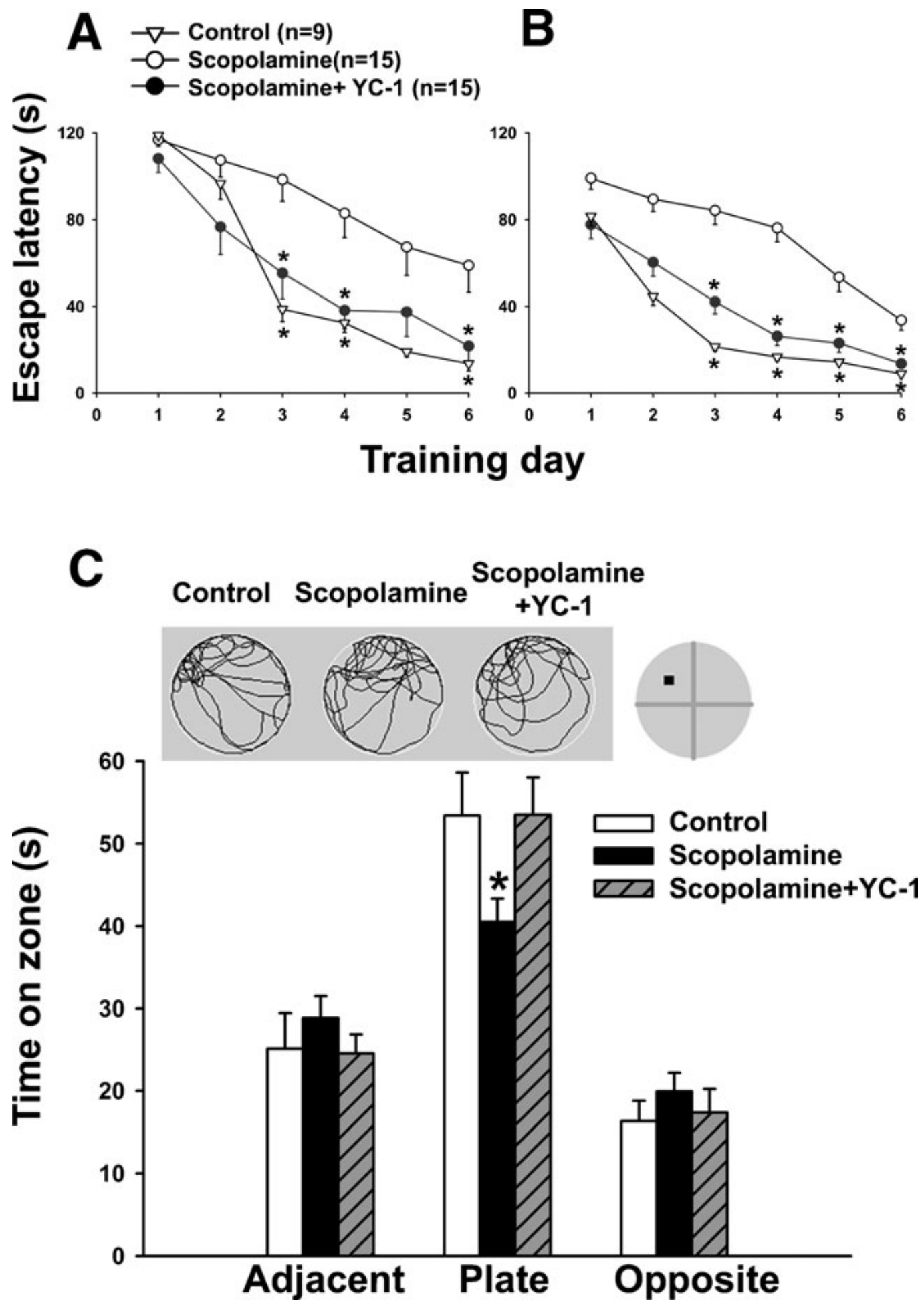

FIG. 8. Effect of YC-1 on scopolamine-induced impairment on Morris water maze task. Both scopolamine (i.p., $0.2 \mathrm{mg} / \mathrm{kg}$ ) and YC-1 were injected (i.p., $1 \mathrm{mg} / \mathrm{kg}$ ) $20 \mathrm{~min}$ before the first trial of each daily training session. Note that scopolamine impaired the learning behaviour and YC-1 reversed the impairment induced by scopolamine both on the first trial (A) and the mean value of the four trials (B). The probe test was examined on day 7 and YC-1 antagonized the decrease of the time on plate zone caused by scopolamine (C). Data are presented as mean \pm SEM $(n=15$ for each). $* P<0.05$ compared with control using twoway ANOVA followed by the post hoc Scheffe test.

Scopolamine is a drug commonly used in the study of memory. It blocks the muscarinic cholinergic receptors, which are involved in learning and memory. In animal studies, scopolamine administration has been used as a simple method to induce cholinergic deficit, which produces impairments on a wide range of learning and memory tasks (Sitaram et al., 1928; Rubaj et al., 2003). Our studies show that YC-1 ameliorates scopolamine-induced impairment of learning behaviours in both Morris water maze performance and passive avoidance task. 


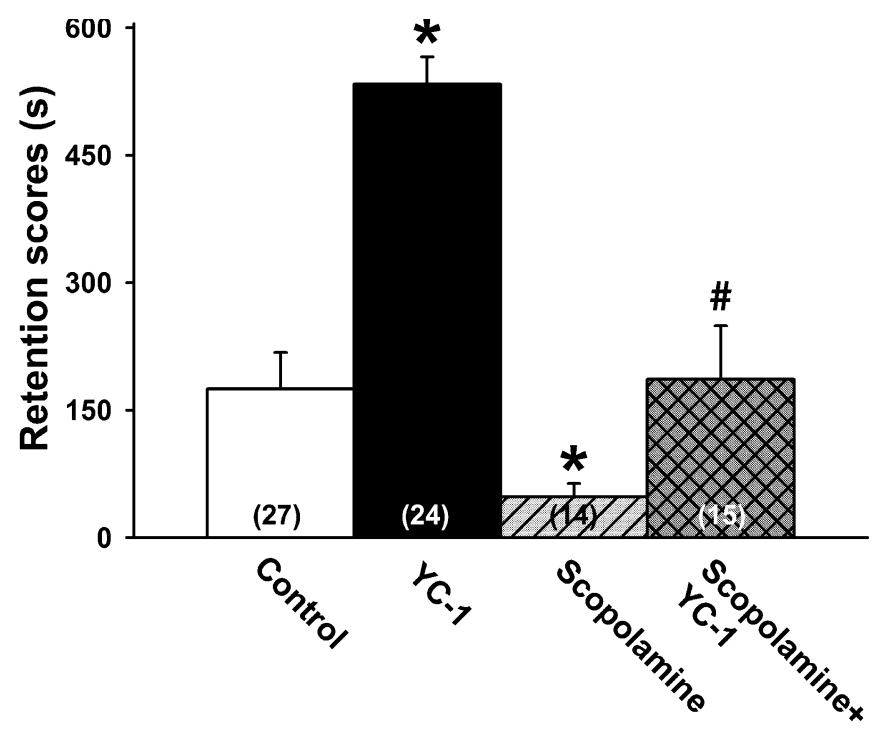

FIG. 9. Effect of YC-1 on scopolamine-induced impairment on passive avoidance task. Passive avoidance was assessed on day 2 after training by measuring the latency for re-entering a chamber previously paired with footshock. Scopolamine decreased the retention scores and rats received both scopolamine $(0.2 \mathrm{mg} / \mathrm{kg}$, i.p. $)$ and YC-1 $(1 \mathrm{mg} / \mathrm{kg}$, i.p. $) 20 \mathrm{~min}$ before footshock markedly prolonged the retention latency tested on day 2. Data are presented as mean \pm SEM $(n) . * P<0.05$ compared with control using nonparametric Kruskal-Wallis followed by the Mann-Whitney $U$-test. $\# P<0.05$ compared with scopolamine-treated group.

These results suggest that YC-1 may be effective for therapy in patients with a dementia such as Alzheimer's disease.

In conclusion, $\mathrm{YC}$-1-like substances are intriguing candidates to act as potent modulators of cGMP-mediated effects within the central nervous system. Our results show that YC-1 improves the formation of new memory and reverses the scopolamine-induced memory deficits in different learning tasks. The remarkable characteristics of YC-1 in potentiating NO-stimulated guanylate cyclase activity during the induction of activity-dependent synaptic plasticity and the high spatial and temporal specificity of NO-induced cellular actions warrant that YC-1 will enhance acquisition of new memory and suggest that YC-1 might be a good candidate for new therapeutic drugs aimed at improving learning and memory in humans.

\section{Acknowledgements}

This work was supported by research grants from the National Science Council.

\section{Abbreviations}

ACSF, artificial cerebrospinal fluid; CMC, carboxymethylcellulose; KT5823, (9S, 10R，12R)-2,3,9,10,11,12, hexahydro-10-methoxy-2,9-dimethyl-1-ox09.12-epoxy-1H-diindolo[1,2,3-fg:3',2',1'-kl]pyrrolo[3,4-1]1,6]benzodiazocine10-carboxylic acid methyl ester; i.c.v., intracerebroventricular; i.p., intraperitoneal; L-NAME, $\mathrm{N}^{\mathrm{G}}$-nitro-L-arginine-methylester; LTD, long-term depression; LTP, long-term potentiation; NMDA, $N$-methyl-D-aspartate; NO, nitric oxide; NOS, NO synthase; ODQ, $1 \mathrm{H}-[1,2,4]$-oxadiazolo(4,3-a)-quinoxalin-1-one; PKG, cGMP-dependent protein kinase; Rp-8-Br-PET-cGMPS, $\beta$-phenyl-1,N2etheno-guanosine $3^{\prime}, 5^{\prime}$-monophosphorothioate; sGC, soluble guanylate cyclase; YC-1, [3-(5-hydroxymethyl-2-furyl)-1-benzyl-indazole].

\section{References}

Abel, T. \& Lattal, K.M. (2001) Molecular mechanisms of memory acquisition, consolidation and retrieval. Curr. Opin. Neurobiol., 11, 180-187.
Aprikyan, G.V. \& Gekchyan, K.G. (1988) Release of neurotransmitter amino acids from rat brain synaptosomes and its regulation in aging. Gerontology, 34, 35-40.

Barnes, C.A. (1988) Spatial learning and memory processes: the search for their neurobiological mechanisms in the rat. Trends Neurosci., 11, 163169.

Bernabeu, R., de Stein, M.L., Fin, C., Izquierdo, I. \& Medina, J.H. (1995) Role of hippocampal $\mathrm{NO}$ in the acquisition and consolidation of inhibitory avoidance learning. Neuroreport, 6, 1498-1500.

Bolshakov, V.Y., Golan, H., Kandel, E.R. \& Siegelbaum, S.A. (1997) Recritment of new sites of synaptic transmission during the cAMPdependent late phase of LTP at CA3-CA1 synapses in the hippocampus. Neuron, 1, 635-651.

Bon, C.L.M. \& Garthwaite, J. (2003) On the role of nitric oxide in hippocampal long-term potentiation. J. Neurosci., 23, 1941-1948.

Boulton, C.L., Southam, E. \& Garthwaite, J. (1995) Nitric oxide-dependent long-term potentiation is blocked by inhibitors of soluble guanylyl cyclase. Neuroscience, 69, 699-703.

Bourtchouladze, R., Frenguelli, B., Blendy, J., Cioffi, D., Schutz, G. \& Silva, A.J. (1994) Deficient long-term memory in mice with a targeted mutation of the cAMP-responsive element-binding protein. Cell, 79, 59-68.

Cahill, L. \& McGaugh, J.L. (1990) Amygdaloid complex lesions differentially affect retention of tasks using appetitive and aversive reinforcement. Behav. Neurosci., 104, 532-543.

Carew, T.J. \& Sutton, M.A. (2001) Molecular stepping stones in memory consolidation. Nature Neurosci., 4, 769-771.

Chien, W.L., Liang, K.C., Teng, C.M., Kuo, S.C., Lee, F.Y. \& Fu, W.M. (2003) Enhancement of long-term potentiation by a potent nitric oxide-guanylyl cyclase activator, 3-(5-hydroxymethyl-2-furyl)-1-benzyl-indazole. Mol. Pharmacol., 63, 1322-1328.

Daniel, H., Levenes, C. \& Crepel, F. (1998) Cellular mechanisms of cerebellar LTD. Trends Neurosci., 21, 401-407.

Friebe, A., Mullershausen, F., Smolenski, A., Walter, U., Schultz, G. \& Koesling, D. (1998) YC-1 potentiates nitric oxide- and carbon monoxideinduced cyclic GMP effects in human platelets. Mol. Pharmacol., 54, 962967.

Friebe, A., Schultz, G. \& Koesling, D. (1996) Sensitizing soluble guanylyl cyclase to become a highly CO-sensitive enzyme. EMBO J., 15, 6863-6868.

Galle, J., Zabel, U., Hübner, U., Hatzelmann, A., Wagner, B., Wanner, C. \& Schmidt, H.H.H.W. (1999) Effects of the soluble guanylyl cyclase activator, YC-1, on vascular tone, cyclic GMP levels and phosphodiesterase activity. Br. J. Pharmacol., 127, 195-203.

Gilbert, D.B., Patterson, T.A. \& Rose, S.P. (1991) Dissociation of brain sites necessary for registration and storage of memory for one-trial passive avoidance task in the chick. Behav. Neurosci., 105, 553-561.

Gudi, T., Huvar, I., Meinecke, M., Lohmann, S.M., Boss, G.R. \& Pilz, R.B. (1996) Regulation of gene expression by cGMP-dependent protein kinase. J. Biol. Chem., 271, 4597-4600.

Haley, J.E., Wilcox, G.L. \& Chapman, P.F. (1992) The role of nitric oxide in hippocampal long-term potentiation. Neuron, 8, 211-216.

Hawkins, R.D. (1996) NO honey, I don't remember. Neuron, 16, 465-467.

Hawkins, R.D., Kandel, E.R. \& Siegelbaum, S.A. (1993) Learning to modulate transmitter release: themes and variations in synaptic plasticity. Annu. Rev. Neurosci., 16, 625-665.

Hawkins, R.D., Son, H. \& Arancio, O. (1998) Nitric oxide as a retrograde messenger during long-term potentiation in hippocampus. Prog. Brain Res., 118, $155-172$.

Ito, M. (2000) Mechanisms of motor learning in the cerebellum. Brain Res., 886, 237-245.

Ito, M. \& Karachot, L. (1990) Messengers mediating long-term desensitization in cerebellar purkinje cells. Neuroreport, 1, 129-132.

Ko, F.N., Wu, C.C., Kuo, S.C., Lee, F.Y. \& Teng, C.M. (1994) YC-1, a novel activator of platelet guanylate cyclase. Blood, 84, 4226-4233.

Lancaster, J.R. (1997) A tutorial on the diffusibility and reactivity of free nitric oxide. Nitric Oxide, 1, 18-30.

Li, J., Smith, S.S. \& McElligott, J.G. (1995) Cerebellar nitric oxide is necessary for vestibulo-ocular reflex adaptation, a sensorimotor model of learning. J. Neurophysiol., 74, 489-494.

Liang, K.C., Tsui, K.Y., Tyan, Y.M. \& Chiang, T.C. (1998) Buspirone impaired acquisition and retention in avoidance tasks: involvement of the hippocampus. Chin. J. Physiol., 41, 33-44.

Malenka, R.C. \& Nicoll, R.A. (1999) Long-term potentiation-a decade of progress? Science, 285, 1870-1874.

Mulsch, A., Bauersachs, J., Schafer, A., Stasch, J.P., Kast, R. \& Busse, R. (1997) Effect of YC-1, an NO-independent, superoxide-sensitive stimulator 
of soluble guanylyl cyclase, on smooth muscle responsiveness to nitrovasodilators. Br. J. Pharmacol., 120, 681-689.

Palmer, A.M. (1996) Neurochemical studies of Alzheimer's disease. Neurodegeneration, 5, 381-391.

Paxinos, G. \& Watson, C. (1997) The rat brain in stereotaxic coordinates. 3rd Compact Edn. Academic Press, San Diego.

Perkel, D.J., Petrozzino, J.J., Nicoll, R.A. \& Connor, J.A. (1993) The role of $\mathrm{Ca}^{2+}$ entry via spatially activated NMDA receptors in the induction of longterm potentiation. Neuron, 11, 817-823.

Prast, H. \& Philippu, A. (2001) Nitric oxide as modulator of neuronal function. Prog. Neurobiol., 64, 51-68.

Rubaj, A., Zgodzinski, W. \& Sieklucka-Dziula, M. (2003) The influence of adenosine A3 receptor agonist: IB-MECA, on scopolamine- and MK-801induced memory impairment. Behav. Brain Res., 141, 11-17.

Schafe, G.E., Nader, K., Blair, H.T. \& LeDoux, J.E. (2001) Memory consolidation of Pavlovian fear conditioning: a cellular and molecular perspective. Trends Neurosci., 24, 540-546.

Shibuki, K. \& Okada, D. (1991) Endogenous nitric oxide release required for long-term synaptic depression in the cerebellum. Nature, 349, 326-328.

Shors, T.J. \& Matzel, L.D. (1997) Long-term potentiation: What's learning got to do with it? Behav. Brain Sci., 20, 597-614.
Sitaram, N., Weingartner, H. \& Gillin, J.C. (1928) Human serial learning enhancement with arecholine and choline impairment with scopolamine. Science, 211, 274-276.

Son, H., Hawkins, R.D., Martin, K., Kiebler, M., Huang, P.L., Fishman, M.C. \& Kandel, E.R. (1996) Long-term potentiation is reduced in mice that are doubly mutant in endothelial and neuronal nitric oxide synthase. Cell, 87, 1015-1023.

Telegdy, G. \& Kokavszky, R. (1997) The role of nitric oxide in passive avoidance learning. Neuropharmacology, 36, 1583-1587.

Tsien, J.Z., Huerta, P.T. \& Tonegawa, S. (1996) The essential role of hippocampal CA1 NMDA receptor-dependent synaptic plasticity in spatial learning. Cell, 87, 1327-1338.

Watanabe, Y., Saito, H. \& Abe, K. (1995) Nitric oxide is involved in long-term potentiation in the medial but not lateral amygdala neuron synapses in vitro. Brain Res., 688, 233-236.

Wilson, R.I., Yanovsky, J., Godecke, A., Stevens, D.R., Schrader, J. \& Haas, H.L. (1997) Endothelial nitric oxide synthase and LTP. Nature, 386, 338.

Zhuo, M., Hu, Y., Schultz, C., Kandel, E.R. \& Hawkins, R.D. (1994) Role of guanylyl cyclase and cGMP-dependent protein kinases in long-term potentiation. Nature, 368, 635-639. 